Trauma Berufskrankh 2014 • 16[Suppl 1]:76-80 DOI 10.1007/s10039-013-1975-0

Online publiziert: 28. Juni 2013

(c) Springer-Verlag Berlin Heidelberg 2013

M. Dudda - T. Fehmer · T.A. Schildhauer · C. Kruppa

Chirurgische Klinik und Poliklinik , BG-Universitätsklinikum Bergmannsheil, Bochum

\title{
Kindliche post-
} traumatische proximale radioulnare Synostose

In der existierenden Literatur sind posttraumatische radioulnare Synostosen in der Regel im Rahmen von Fallbeispielen und kleineren Fallbeispielserien beschrieben [6, 31, 36]. Vince u. Miller [36] analysierten 198710 Kinder mit radioulnaren Synostosen nach Unterarmfrakturen oder -osteotomien. Dieser Veröffentlichung ging eine Studie zu Synostosen im Erwachsenenalter voraus [35]. Die Autoren beschrieben eine Inzidenz von $2 \%$ in einer Population von 2318 Erwachsenen mit Unterarmfrakturen und entwickelten ein auf der Lokalisation der Synostose beruhendes Klassifikationssystem aus $3 \mathrm{Ty}-$ pen [35]:

- Typ 1 (distaler Typ) ist dem distalen intraartikulären Bereich des Unterarms zugeordnet,

- Typ 2 (diaphysär) dem mittleren extraartikulären und

- Typ 3 (proximal) dem proximalen Unterarmdrittel.

Jupiter u. Ring [15] entwickelten zusätzlich eine Subklassifikation der nach Vince u. Miller [35] als Typ 3 klassifizierten Synostose. Sie beschrieben

- einen Typ A, lokalisiert distal der Tuberositas radii,

- einen Typ B im Bereich des radioulnaren Gelenks und

- einen Typ C mit additiver Ankylose des Ellenbogengelenks mit zusätzlichen humeroulnaren Ossifikationen [15].

\section{Therapieempfehlungen}

In der Literatur existiert derzeit keine standardisierte Behandlungsmethode. Mehrere operative Verfahren mit und ohne Interpositionstechniken sind beschrieben $[1,15,16,29,36,38]$. Der richtige Zeitpunkt der Behandlung stellt ebenfalls einen wichtigen Diskussionspunkt dar. Vince u. Miller [36] beschrieben einerseits erhöhte Rezidivraten nach früher Synostosenresektion, andererseits kann eine verspätete Resektion durch Vernarbungen und osteophytäre Knochendeformitäten erschwert werden.

Aufgrund von fehlenden Behandlungsempfehlungen und der begrenzten Literatur werteten wir alle Kinder und Jugendlichen mit posttraumatischer Synostose, die innerhalb der letzten 15 Jahre in unserer Klinik operativ behandelt wurden, aus, um eine Empfehlung hinsichtlich des Behandlungszeitpunkts und der operativen Technik zu erarbeiten.

\section{Klinische Ergebnisse}

\section{Patienten}

Unser Patientengut umfasste 8 operativ behandelte Patienten mit posttraumatischen Synostosen im Bereich des proximalen Unterarms (Typ 3 nach Vince u. Miller [36]). Ursache der radioulnaren Synostose waren Radiusköpfchen- bzw. -halsfrakturen (5 Kinder), und proxima- 

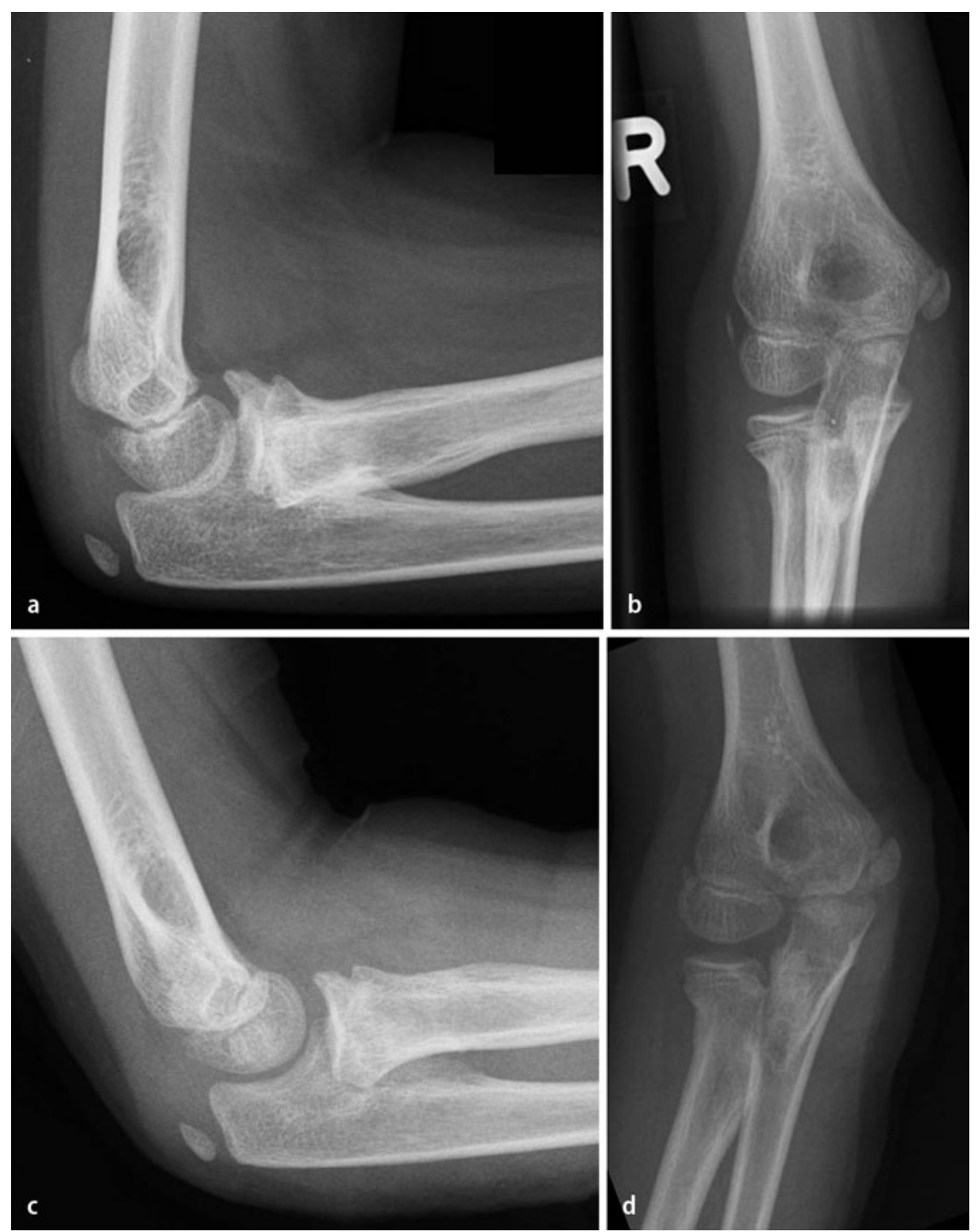

Abb. $1 \Delta$ Proximale radioulnare Synostose bei einem 10-jährigen Jungen nach Radiushalsfraktur $(\mathbf{a}, \mathbf{b})$, Resektion der Synostose 10 Monate nach dem initialen Trauma $(\mathbf{c}, \mathbf{d})$

le Unterarmfrakturen (3 Kinder). Bei den Patienten handelte es sich um 4 Mädchen und 4 Jungen.

Das Durchschnittsalter der Kinder zum Zeitpunkt der Fraktur betrug 9,0 02,56 Jahre ( 6 bis 14 Jahre) und zum Zeitpunkt der operativen Therapie $11,9 \pm 3,09$ Jahre (7 bis 16 Jahre).

\section{Art und Zeitpunkt der Therapie}

Bei 5 Patienten erfolgte die Resektion der Synostose ohne Interpositionsverfahren, 2-mal mit gleichzeitiger Arthrolyse des Ellenbogengelenks und 1-mal mit Interposition eines lokalen Faszienlappens

(• Abb. 1).

\section{Resultate}

Bei 2 Kindern schlug die primäre Therapie der Synostose fehl. Bei dem Patienten mit der längsten zeitlichen Spanne zwischen dem Trauma und der Resektion der Synostose (5 Jahre) erfolgte schließlich die Radiusköpfchenresektion. Einmalig wurde auf Wunsch der Eltern keine operative Therapie einer Rezidivsynostose durchgeführt. Bei dem Patienten mit Interposition des lokalen Faszienlappens mussten im Verlauf (1 Monat nach der Operation) eine Narkosemobilisation und Physiotherapie mittels Regionalanästhesie durchgeführt werden.

Eine Verbesserung des Bewegungsausmaßes im Vergleich zur präoperativen eingesteiften Situation konnte bei allen Patienten beobachtet werden. Das durchschnittliche Bewegungsausmaß für Pronation/Supination betrug postoperativ $36^{\circ} / 0^{\circ} / 53^{\circ}$. Jedoch kam es bei allen Patienten zu einem postoperativen Verlust des intraoperativ erlangten Bewegungsausmaßes.

\section{Diskussion}

Die proximale radioulnare Synostose ist selten. Sie stellt eine ernsthafte Komplikation nach Radiusköpfchen- bzw. -halsfrakturen $[7,22,25,33,34]$, aber auch nach Radiusköpfchenluxationen oder Monteggia-Frakturen dar und ist nach operativer Behandlung von deutlich dislozierten Frakturen beschrieben $[3,5,19,28]$. Neben Dislokationen wurden auch periostale Interposition [26], Operationstraumen $[4,10]$ sowie wiederholte Manipulation [31] in der Literatur als Ursache genannt.

Newman [25] beobachtete ein Auftreten in $10 \%$ seiner Fälle. Nenopoulus et al. [24] wiederum beschrieben in ihrer aus 45 Kindern bestehenden Studienpopulation eine Inzidenz von 9\% mit Ausbildung nach dislozierten Frakturen. Vocke u. von Laer [37] berichteten in ihrer Publikation, dass es nach Radiusköpfchenfrakturen in 1 von 38 Fällen zur Ausbildung einer Synostose kam. Häßle u. Mellerowicz [9] analysierten 116 Frakturen des proximalen Unterarms im Kindesalter und konnten die Ausbildung von 2 radioulnaren Synostosen beobachten. Sie berichteten, dass in allen Fällen eine Seitdis- 
lokation des Radiusköpfchens bzw. -halses zwischen 2 und $5 \mathrm{~mm}$ vorlag. Ähnliches wurde auch von Newman [25] beobachtet, in 4 von 5 Fällen, in denen es zur Ausbildung einer Synostose kam, lag eine Seitdislokation von mehr als $2 \mathrm{~mm}$ vor.

Ebenso wie die Ursache der Synostenausbildung nicht endgültig geklärt ist, besteht auch keine Einigkeit über die richtige Art sowie den korrekten Zeitpunkt der Behandlung. Im Erwachsenenalter empfahlen Vince u. Miller [35] die Synostosenentfernung nach Ablauf 1 Jahres nach Verletzung, um den Abschluss der Kallusformation abzuwarten, im Kindesalter warnten sie jedoch vor einer zu späten Resektion, da durch Vernarbungen und Kontrakturen bei lange bestehender Synostose eine Wiedergewinnung des Bewegungsausmaßes erschwert sein kann. Im Gegensatz dazu empfahl Ogden [27] die Resektion im Lauf der ersten 6 Monate.

In unserem Patientengut betrug der Zeitraum bis zur Entfernung der Synostose nach dem Trauma in 5 Fällen zwischen 5 und 30 Monate. Alle Patienten, bei denen die Operation im Intervall von weniger als 30 Monaten nach dem Trauma erfolgte, wurden durch Resektion der Synostose behandelt, und die Ergebnisse waren akzeptabel, sodass wir uns der Empfehlung von Vince u. Miller [36] anschließen.

In 2 von 3 Fällen, bei denen die primäre Therapie der Synostose erst mehr als 5 Jahre nach dem Trauma durchgeführt wurde, erfolgte primär die Radiusköpfchenresektion aufgrund von deutlichen Osteophyten, Weichteilvernarbungen und einer vorangeschrittenen Ankylose des Ellenbogengelenks. In beiden Fällen kam es nicht zur Rezidivausbildung. Im 3. Fall wurde primär nur die Synostose reseziert, es kam jedoch zum Rezidiv, weshalb dann auch in diesem Fall das Radiusköpfchen reseziert wurde.

Merke. Die Radiusköpfchenresektion im Kindes- und Jugendalter sollte als SalvageProzedur angesehen und nur nach sorgfältiger Indikationsstellung in Erwägung gezogen werden.

Die Behandlungsmöglichkeiten der radioulnaren Synostose sind in der Regel in Fallbeispielen oder kleinen Fallserien beschrieben $[1,17,21,29,36]$. Aner et al. [1]

Trauma Berufskrankh 2014 - 16[Suppl 1]:76-80 DOI 10.1007/s10039-013-1975-0

๑) Springer-Verlag Berlin Heidelberg 2013

\section{Dudda $\cdot$ T. Fehmer $\cdot$ T.A. Schildhauer $\cdot$ C. Kruppa Kindliche posttraumatische proximale radioulnare Synostose}

\section{Zusammenfassung}

Hintergrund. Die kindliche posttraumatische proximale radioulnare Synostose ist eine seltene, aber trotzdem nicht zu unterschätzende Komplikation von Radiushals- bzw. -köpfchen- und proximalen Unterarmfrakturen sowie Ellenbogenluxationen. Ein einheitliches Behandlungsschema existiert bis heute nicht, und es gibt zahlreiche operative Behandlungsmethoden mit und ohne Interpositionsverfahren.

Therapieverfahren und -zeitpunkt. Die Art sowie der Zeitpunkt der Behandlung der Synostose werden nach wie vor kontrovers diskutiert. Wir empfehlen, nach Auswertung unserer eigenen Daten und der Literatur, eine Resektion der Synostose in den ersten 6 bis 24 Monaten nach Auftreten, ohne zwingendes Interpositionsverfahren. Ausgehend von einer fehlenden Beweglichkeit für Supination/Pronation konnte das Bewegungsausmaß in unserem Patientengut verbessert werden. Bei verzögerter Diagnosestellung erst mehrere Jahre nach dem Trauma - kann eine Radiusköpfchenresektion als SalvageProzedur, bei meist sehr kontrakten Weichteilen, durchgeführt werden, wobei die Ergebnisse nicht zufriedenstellend sind.

\section{Schlüsselwörter}

Knochenfraktur $\cdot$ Radius $\cdot$ Ulna $\cdot$ Synostose Komplikation

\section{Pediatric posttraumatic proximal radio-ulnar synostosis}

\section{Abstract}

Background. The posttraumatic proximal cross-union of the forearm in childhood is a rare complication after radial head, neck or proximal forearm fractures and elbow dislocations. No standardized treatment exists. Several operative procedures with and without interposition techniques have been described in the literature.

Therapy procedure and timing. There is still a controversy about the best procedure and the timing of an operative treatment regarding the cross-union in childhood. Many procedures and treatment options have been described in case reports and small case series. After analysis of the data and review of the literature the recommended procedure is resection of the cross-union within 6-24 months after occurrence without necessarily using an interposition technique. On average the range of motion was improved starting from an entirely neutral stiffness for pronation and supination. If the cross-union has existed for several years with ankylosis of the elbow and bony deformities of the proximal radius, an excision of the radial head as salvage procedure can be accomplished but the results are not satisfying.

\section{Keywords}

Fractures, Bone · Radius · Ulna · Synostosis . Complication berichteten über 2 Fälle radioulnarer Synostosen. Einmal wurde die Resektion ohne Interponat, 33 Monate nach initialer Frakturversorgung, mit einem resultierenden Bewegungsausmaß von $45^{\circ} / 0^{\circ} / 70^{\circ}$ Pronation/Supination, durchgeführt. Im zweiten Fall erfolgte bei einer Typ-2-Synostose nach Vince u. Miller [36] die Resektion 8 Monate nach initialer Versorgung mittels Interposition eines GORE-TEX ${ }^{\circledR}$ Vascular Grafts um die Ulna als experimentelles Vorgehen. Hiermit konnte ein Bewegungsausmaß von $80^{\circ} / 0^{\circ} / 90^{\circ}$ erreicht werden. Die Autoren berichteten ein ansteigendes Risiko für die Ausbildung von Synostosen nach dem Alter von 10 Jah- ren und bei ausgeprägter Frakturdislokation [1]. Wierer et al. [38] wendeten die Methode von Kamineni et al. [16] mittels Segmentresektion von $1 \mathrm{~cm}$ des Knochens (des Radius unter Belassen der Synostose) in einem Fallbeispiel mit gutem Ergebnis an. Auch die Behandlung mittels Radiusköpfchenresektion als Salvage-Prozedur ist in der Literatur mit guten Ergebnissen beschrieben [11, 12].

Die Interposition von freien vaskularisierten Faszienfettlappen [17] oder Silikonmembranen [29] nach Synostosenresektion ist publiziert. Von Laer [20] erwähnte 2004, dass eine erfolgreiche Behandlung von posttraumatischen Synos- 
tosen wahrscheinlicher ist als die von kongenitalen Synostosen und dass die Interposition von vaskularisierten Transplantaten vielversprechend sei.

Im Falle der Synostosenresektion mit Interposition eines lokalen Faszienlappens erfolgte in unserem Patientengut 1 Monat nach der Operation die Narkosemobilisation bei zunehmender Bewegungseinschränkung. Dies war aber aller Wahrscheinlichkeit nach auf eine unzureichende Physiotherapie zurückzuführen. Die postoperative Nachbehandlung stellt, neben dem Zeitpunkt der Resektion und der operativen Technik, daher einen wichtigen und bisher wenig untersuchten Punkt dar. Insbesondere da alle Patienten postoperativ eine Abnahme des intraoperativ erzielten Bewegungsausmaßes zeigten, sind unserer Ansicht nach die intensive krankengymnastische Nachbehandlung und engmaschige Kontrolle der Kinder zur Überprüfung des Therapieerfolgs unabdingbar. Diese Patienten sollten engmaschig nachuntersucht und an die Klinik gebunden werden.

Merke. Die postoperative intensive Physiotherapie und engmaschige Kontrolle sind unverzichtbar, um einem Verlust des Bewegungsausmaßes vorzubeugen.

Diese Einschätzung wird unserer Meinung nach durch die Tatsache bekräftigt, dass alle Kinder auch ohne radiologisches Synostosenrezidiv postoperativ einen Verlust der Beweglichkeit erlitten, was am ehesten auf Weichteilkontrakturen zurückzuführen ist und somit initial intensiv physiotherapeutisch angegangen werden sollte.

Wir führten zusätzlich die medikamentöse Rezidivprophylaxe mittels eines Antiphlogistikums, wie auch von anderen Autoren in der Literatur beschrieben $[30,32]$, durch, wobei dies nicht evident ist und in der Literatur gerade in Bezug auf Kinder hierzu keine Aussage getroffen wird. Die Bestrahlung wird ebenfalls kontrovers diskutiert $[2,18]$, wobei diese bei verzögert diagnostizierten und behandelten Synostosen mit osteophytären Ausziehungen und Ankylosen in Einzelfällen angewendet wurde. Dies geht allerdings nur auf Daten aus der Behandlung von periartikulären Ossifikationen bei Er- wachsenen oder Schädel-Hirn-Verletzter zurück $[2,13,14,18]$.

\section{Resümee}

Zusammenfassend zeigen unsere Ergebnisse, dass die Behandlung der Typ-3Synostose eine weiterhin schwierige Aufgabe darstellt, deren Ergebnisse nicht zufriedenstellend sind. In unserem Patientengut konnte in 7 von 8 Fällen ohne $\mathrm{Zu-}$ hilfenahme der oben genannten Interponate eine durchschnittliche Beweglichkeit von etwa $40^{\circ} / 0^{\circ} / 50^{\circ}$ für Pronation/Supination erreicht werden.

Eine sehr lange zeitliche Verzögerung kann unserer Meinung nach, ähnlich wie bei Vince u. Miller [36] erwähnt, zu Vernarbungen des Weichteilgewebes führen und eine Wiedergewinnung des Bewegungsausmaßes erschweren. Unsere Ergebnisse unterstützen diese Erfahrungen, denn insbesondere die sehr späten Behandlungen in unserer Patientengruppe waren alle von Radiusköpfchenresektionen gefolgt.

Bei verzögert diagnostizierter Synostose und sehr späten Resektionen sowie bei Vorliegen eines ankylotischen Ellenbogengelenks kann die Radiusköpfchenresektion mit oder ohne Arthrolyse eine Salvage-Prozedur darstellen und zu akzeptablen Ergebnissen führen, wobei die hiermit erreichte durchschnittliche Bewegung in unserem Kollektiv nicht zufriedenstellend war.

\section{Fazit für die Praxis}

- Die Resektion der posttraumatischen proximalen radioulnaren Synostose sollte im Intervall zwischen 6 und 24 Monaten erfolgen, da dabei anscheinend die besten Ergebnisse erzielt werden können.

- Eine intensive und engmaschige Physiotherapie und Nachuntersuchung sind dringend zu empfehlen, um die intraoperativ erzielte Beweglichkeit aufrechtzuerhalten.

\section{Korrespondenzadresse}

\section{Dr. M. Dudda}

Chirurgische Klinik und Poliklinik, BG-Universitätsklinikum Bergmannsheil, Bürkle-de-la-Camp Platz 1, 44789 Bochum marcel.dudda@ruhr-uni-bochum.de

Interessenkonflikt. Der korrespondierende Autor gibt für sich und seine Koautoren an, dass kein Interessenkonflikt besteht.

The supplement containing this article is not sponsored by industry.

\section{Literatur}

1. Aner A, Singer M, Feldbrin Z et al (2002) Surgical treatment of posttraumatic radioulnar synostosis in children. J Pediatr Orthop 22(5):598-600

2. Cullen JP, Pellegrini VD Jr, Miller RJ, Jones JA (1994) Treatment of traumatic radioulnar synostosis by excision and postoperative low-dose irradiation. J Hand Surg Am 19(3):394-401

3. Degreef I, De Smet L (2004) Missed radial head dislocations in children associated with ulnar deformation: treatment by open reduction and ulnar osteotomy. J Orthop Trauma 18(6):375-378

4. Dougall AJ (1969) Severe fracture of the neck of the radius in children. J $R$ Coll Surg Edinb 14(4):220-225

5. Exner GU (2001) Missed chronic anterior Monteggia lesion. Closed reduction by gradual lengthening and angulation of the ulna. J Bone Joint Surg $\mathrm{Br}$ 83(4):547-550

6. Fielding JW (1964) Radio-ulnar crossed union following displacement of the proximal radial epiphysis. A case report. J Bone Joint Surg Am 46:1277-1278

7. Gao GX, Zhang RY (1984) Radial neck fracture in children. Chin Med J 97(12):893-896

8. Gross SD (1864) A system of surgery, 3. Aufl. Blanchard \& Lea, Philadelphia, S 916

9. Häßle M, Mellerowicz H (1991) Frakturen des proximalen Radius im Wachtumsalter. Unfallchirurgie 17:24-33

10. Henrikson B (1969) Isolated fractures of the proximal end of the radius in children epidemiology, treatment and prognosis. Acta Orthop Scand 40(2):246-260

11. Herbertsson $P$, Josefsson $P O$, Hasserius $R$ et al (2004) Fractures of the radial head and neck treated with radial head excision. J Bone Joint Surg Am 86-A(9):1925-1930

12. Hresko MT, Rosenberg BN, Pappas AM (1999) Excision of the radial head in patients younger than 18 years. J Pediatr Orthop 19(1):106-113

13. Ippolito E, Formisano R, Farsetti P et al (1999) Excision for the treatment of periarticular ossification of the knee in patients who have a traumatic brain injury. J Bone Joint Surg Am 81(6):783-789

14. Ippolito E, Formisano R, Caterini R et al (1999) Operative treatment of heterotopic hip ossification in patients with coma after brain injury. Clin Orthop Relat Res 365:130-138

15. Jupiter JB, Ring D (1998) Operative treatment of post-traumatic proximal radioulnar synostosis. J Bone Joint Surg Am 80(2):248-257 
16. Kamineni S, Maritz NG, Morrey BF (2002) Proximal radial resection for posttraumatic radioulnar synostosis: a new technique to improve forearm rotation. J Bone Joint Surg Am 84-A(5):745-751

17. Kanaya F, Ibaraki K (1998) Mobilization of a congenital proximal radioulnar synostosis with use of a free vascularized fascio-fat graft. J Bone Joint Surg Am 80(8):1186-1192

18. Kluger G, Kochs A, Holthausen H (2000) Heterotopic ossification in childhood and adolescence. J Child Neurol 15(6):406-413

19. Koslowsky TC, Mader K, Wulke AP et al (2006) Operative treatment of chronic Monteggia lesion in younger children: a report of three cases. J Shoulder Elbow Surg 15(1):119-121

20. Laer C van (2004) Korrektureingriffe am kindlichen Ober- und Unterarm. Unfallchirurg 107:552-562

21. Laer L von, Pirwitz A, Vocke AK (1997) Posttraumatische Problemfälle am kindlichen Ellbogen. Orthopade 26(12):1030-1036

22. Lindham S, Hugosson C (1979) The significance of associated lesions including dislocation in fractures of the neck of the radius in children. Acta Orthop Scand 50(1):79-83

23. Mouchet A (1900) Les fractures du col du radius. Chirurgie 21:596-622

24. Nenopoulos SP, Beslikas TA, Gigis JP (2009) Longterm follow-up of combined fractures of the proximal radius and ulna during childhood. J Pediatr Orthop B 18(5):252-260

25. Newman JH (1977) Displaced radial neck fractures in children. Injury 9(2):114-121

26. O凶Brien PI (1965) Injuries involving the proximal radial epiphysis. Clin Orthop Relat Res 41:51-58

27. Ogden JA (1982) Skeletal injury in the child. Lea \& Febiger, Philadelphia

28. Powell RS, Bowe JA (2002) Ipsilateral supracondylar humerus fracture and Monteggia lesion: a case report. J Orthop Trauma 16(10):737-740

29. Proubasta IR, Lluch A (1995) Proximal radio-ulnar synostosis treated by interpositional silicone arthroplasty. A case report. Int Orthop 19(4):242244

30. Ritter M, Giobe T (1979) The effect of indomethacin on heterotopic ossification of the elbow following long term coma. J Bone Joint Surg Am 61:113-117

31. Roy DR (1986) Radioulnar synostosis following proximal radial fracture in child. Orthop Rev 15(2):89-94

32. Sodemann B, Persson PE, Nilsson OS (1988) Prevention of periarticular heterotopic ossification following total hip arthroplasty. Clinical experience with indomethacin and ibuprofen. Arch Orthop Trauma Surg 107(6):329-333

33. Steele JA, Graham HK (1992) Angulated radial neck fractures in children. A prospective study of percutaneous reduction. J Bone Joint Surg $\mathrm{Br}$ 74(5):760-764

34. Vahvanen V, Gripenberg L (1978) Fracture of the radial neck in children. A long-term follow-up study of 43 cases. Acta Orthop Scand 49(1):32-38

35. Vince KG, Miller JE (1987) Cross-union complicating fracture of the forearm. Part I: Adults. J Bone Joint Surg Am 69(5):640-653

36. Vince KG, Miller JE (1987) Cross-union complicating fracture of the forearm. Part II: Children. J Bone Joint Surg Am 69(5):654-661

37. Vocke AK, Laer L von (1998) Displaced fractures of the radial neck in children: long-term results and prognosis of conservative treatment. J Pediatr Orthop B 7(3):217-222
38. Wierer M, Huber-Wagner S, Mutschler W (2012) Posttraumatische proximale radioulnare Synostose. Operationstechnik und Literaturübersicht. Unfallchirurg 115(5):451-456 